\title{
Training university students as vaccination champions to promote vaccination in their multiple identities and help address vaccine hesitancy
}

\author{
Nusayba Ali $^{1}$ iD, Diane Ashiru-Oredope ${ }^{1,2}$ iD, Sudaxshina Murdan ${ }^{1}$ iD \\ ${ }^{1}$ UCL School of Pharmacy, University College London, London, United Kingdom \\ ${ }^{2}$ Antimicrobial Resistance and Healthcare Associated Infection, National Infection Service, Public Health England, United Kingdom
}

\author{
Keywords \\ COVID-19 \\ Hesitancy \\ Misconception \\ Myth \\ Promotion \\ Vaccination \\ Correspondence \\ Dr Sudaxshina Murdan \\ University College London \\ 29-39 Brunswick Square \\ London \\ WC1N 1 AX \\ United Kingdom \\ s.murdan@ucl.ac.uk
}

\begin{abstract}
Introduction: Covid-19 related vaccine hesitancy is a major problem worldwide and it risks delaying the global effort to control the pandemic. Covid-19 vaccine hesitancy is also higher in certain communities. Given that prescriber recommendation and community engagement are two effective ways of addressing vaccine hesitancy, training university students to become vaccination champions could be a way of addressing hesitancy, as the champions engage with their communities in their multiple identities. Aim: This study aims to assess the impact of a pilot project conducted in the UCL School of Pharmacy that could pave a way of integrating vaccination championing in the pharmacy undergraduate curriculum to address vaccine hesitancy. Method: Participants completed a pre-workshop questionnaire, attended an online workshop, conducted vaccination-promoting action/s, and provided evidence via a postworkshop questionnaire. Result: Fifty three students completed the course. The students' vaccination-promoting actions ranged from speaking with vaccine-hesitant family, friends and customers in the pharmacy, to posting on various social media platforms. Post-workshop showed an increase in the knowledge of participants regarding vaccination and a decrease in the belief of vaccine misconceptions. After attending the workshop, participants were more likely to engage with vaccine-hesitant friends, family, strangers and patients. They were also more likely to receive the Covid-19 vaccine for them and for their children.
\end{abstract}

\section{Introduction}

Pharmacies and pharmacists are involved in vaccination in many countries through counselling about and administering vaccines (Poudel et al., 2019). There are a number of calls for their role to be expanded (Ciliberti et al., 2020), for example, to address vaccine hesitancy (Lisenby et al., 2019) and play a key role in the roll-out of mass COVID-19 vaccination (Lee et al., 2020). Pharmacists in the United Kingdom (UK) and pharmacy undergraduates in, for example, the United States of America (USA), are already administering the newly approved COVID-19 vaccines, while in other countries, e.g. Australia, pharmacy undergraduates are working at COVID-19 vaccine clinics to prepare the vaccine for administration by others. Practical training to administer vaccines is embedded in the undergraduate pharmacy curriculum at many universities, for example, in the UK, Canada and Australia (Church et al., 2016; Prescott \& Bernhardi, 2019; Bushell et al., 2020), and in the USA, pharmacy students can administer vaccines to patients following appropriate certification (Bowers et al., 2020). It must be noted, however, that the extent of training and engagement of pharmacy students in immunisation programs varies extensively, depending on the universities, which reflects the global variability in that of registered pharmacists. When given the opportunity to engage in immunisation initiatives, pharmacy students have had a range of positive impacts, from increasing vaccination rates to improving patient knowledge of vaccines and vaccine-preventable diseases (Church et al., 2016). Of particular note is Operation Immunisation - an immunisation education campaign designed to 
increase the public's knowledge of immunisations while raising the number of adults receiving immunisations which was collaboratively developed by the American Pharmacists Association Academy of Student Pharmacists (APhA-ASP) and the Student National Pharmaceutical Association (SNPhA) and which has immunised over a million individuals since its launch in 1997 (APhA).

The research literature on pharmacy students' engagement in immunisation initiatives shows that administration of vaccines by students was the most common student intervention, followed by education and counselling of patients (Church et al., 2016). The students' involvement was related to their roles as future healthcare professionals. The authors believe that widening student involvement in immunisation initiatives outside of their future professional role, to encompass their multiple identities, for example, that of a family member, neighbour, friend, acquaintance, community member, social media 'publisher', a stranger is also important, and is missing from most Pharmacy undergraduate curricula. Similarly, education about vaccine hesitancy so that students gain a more nuanced understanding of the topic is also missing from many pharmacy undergraduate curricula. For example, information about the vaccine hesitancy continuum and of the varied reasons why many people are vaccinehesitant is missing from many Pharmacy undergraduate curricula.

Vaccine hesitancy, referring to delaying acceptance or refusing vaccines despite their availability (WHO, 2014; MacDonald \& Hesitancy, 2015) and an expression of concern or doubt about the value or safety of vaccination (Yaqub et al., 2014), sits in the continuum ranging from active demand for vaccines to complete refusal of all vaccines (Dube et al., 2013; WHO, 2014). It is common (reported in more than 90 countries, at all income levels) and growing (Larson et al., 2014; Lane et al., 2018) to such an extent that in 2019, the World Health Organisation listed vaccine hesitancy as one of the top ten threats to global health. Although not always accompanied by the refusal of vaccination or refusal of all vaccines, vaccine hesitancy can soon lead to vaccine refusal, resulting in clusters of unvaccinated individuals, a loss of herd immunity, and disease outbreaks among the unvaccinated. For example, outbreaks or resurgence of measles, mumps, Haemophilus influenza b, pertussis, and polio have occurred in countries where these diseases had previously been eliminated/controlled (WHO, 2014) reversing the decades of gains in vaccination programs. In the current Covid-19 pandemic, vaccine hesitancy is widely prevalent globally and could delay the control of the pandemic (Lazarus et al., 2020).
Individuals' reasons for vaccine hesitancy vary by vaccines and are highly context-specific and multidimensional, ranging from concerns about vaccine safety and adverse effects to mistrust of doctors/health authorities/government sources, of pharmaceutical companies and scientific research to perceptions of low risk of contracting an illness, of low illness severity, and low vaccine efficacy to fear of needles, vaccine cost, religious beliefs, inconvenience, lack of information and their right to choose (Hanson et al., 2018; Paterson et al., 2018; Aggarwal, 2019; Facciola et al., 2019; Swaney \& Burns, 2019; Palamenghi et al., 2020). Even some healthcare practitioners are hesitant about vaccination for themselves and for their patients, citing reasons such as vaccine safety and adverse effects, their lack of knowledge, confidence, and of time, and vaccine cost (Oscarsson et al., 2011; McRee et al., 2014; Yaqub et al., 2014; Verger et al., 2015; Kwok et al., 2021). For COVID 19-vaccines, the novelty of the vaccines, mistrust of vaccine benefit, speed of vaccine development, worry about adverse effects and unforeseen future negative effects, concerns about commercial profiteering by pharmaceutical companies, preference for natural immunity, location of vaccine development are additional factors contributing to vaccine hesitancy (Goldman et al., 2020; Pogue et al., 2020; Taylor et al., 2020).

The healthcare provider plays a critical role in vaccine hesitancy or acceptance, reflected in their advice being the most common reason for vaccine-hesitant parents changing their minds (Gust et al., 2008) and for people supporting vaccination or conversely people citing a lack of a general Practitioner recommendation for being vaccine-hesitant (Yaqub et al., 2014). It is also known that dialogue-based and personalised communications that are tailored to the audience from a trusted community member are the most effective at addressing vaccine hesitancy (WHO, 2014; Dube et al., 2015; Eskola et al., 2015; Jarrett et al., 2015; Nowak et al., 2015). Consequently, training university students, especially those studying health-related subjects, to become vaccination champions is one way of addressing vaccine hesitancy, as the Champions engage with their communities. Educating future healthcare practitioners to have a nuanced understanding of vaccines and vaccine hesitancy would also better prepare them to confidently reassure vaccine-hesitant patients/parents in their professional capacity (Leask et al., 2014). In addition, such education is likely to increase their own vaccine acceptance, given that lack of knowledge is cited as a reason for vaccine hesitancy in healthcare practitioners (Yaqub et al., 2014).

In this paper, the authors reported on a pilot project to train university students studying pharmacy or pharmaceutical sciences to become vaccination champions. The aim of the work was to conduct and 
assess the impact of a pilot project that could pave the way to the integration of vaccination championing in undergraduate and postgraduate programs, with the ultimate aim of enabling vaccination champions to promote vaccination in their multitude of roles, whenever the opportunity arose to address vaccine hesitancy.

\section{Methods}

The 'Becoming a Vaccination Champion' training course took place in July 2020, during the university summer break following the final year exams. The course was advertised by email (Figure 1) to all students enrolled in the MPharm Pharmacy and MSc. programmes at the University College London (UCL) School of Pharmacy, with the course requirements, which were to (i) complete a pre-workshop questionnaire, (ii) attend the two-hour online workshop, (iii) conduct an action promoting vaccination within a week of the online workshop and (iv) complete a post-workshop questionnaire. Interested participants registered via Eventbrite, and they were sent a link to the workshop.

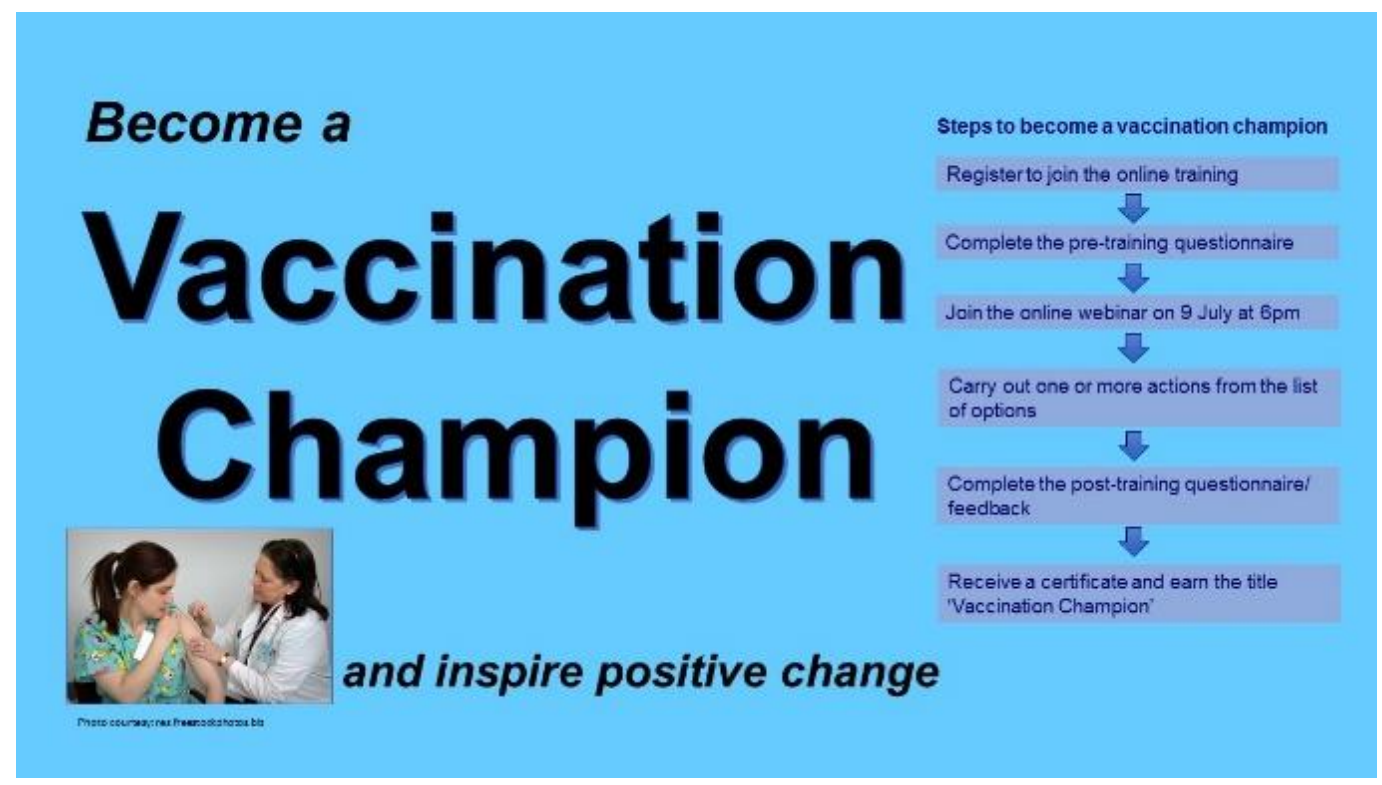

Figure 1: Brochure used in course advertisement

The questionnaire was conducted using Google forms to ascertain the participants' existing knowledge, attitudes, and beliefs about vaccination. Students could choose True/False/l don't know/Prefer not to answer or Extremely unlikely/ Unlikely/ Neutral/Likely/Extremely likely or Strongly disagree/Disagree/ Neither disagree nor agree/Agree/Strongly agree. The questions (shown in Table I) were based on a survey conducted among primary healthcare workers involved in the administration of childhood vaccines in Spain in 2016/2017 (Picchio et al., 2019) but were adapted to reflect the experience of the students completing the pre-training questionnaire. The Spanish survey was used as a starting point for a range of reasons: the survey had the same objectives as the authors' pre-training questionnaire, the respondents in this study (aspiring healthcare professionals) are similar to those who completed the Spanish survey (current health care professionals) and Spain is a high-income country like the UK.
The two-hour online workshop was conducted via zoom and was composed of a presentation that was interspersed with Slido polls and break-out rooms. The authors co-developed the presentation, which was then delivered by the first author, who was, at the time, a second-year MPharm undergraduate. The presentation included the learning outcomes, definition, role of, and the need for vaccination champions, brief explanations of vaccines, vaccination, herd immunity, the direct and indirect benefits of vaccination, vaccine hesitancy, common misconceptions regarding vaccination and the evidence-based scientific counterarguments, and the need to address vaccine hesitancy.

The Slido polls were used to ask the participants the following questions: 1) How confident are you to promote vaccination? 2) What have you learned about vaccination and vaccines that you did not know before? 3) What are the barriers, if any, to you becoming a vaccination champion?, and 5) What action might you take in the next week regarding the next step for you to become a vaccination champion? 
Table I: Pre-workshop and post-workshop survey questions

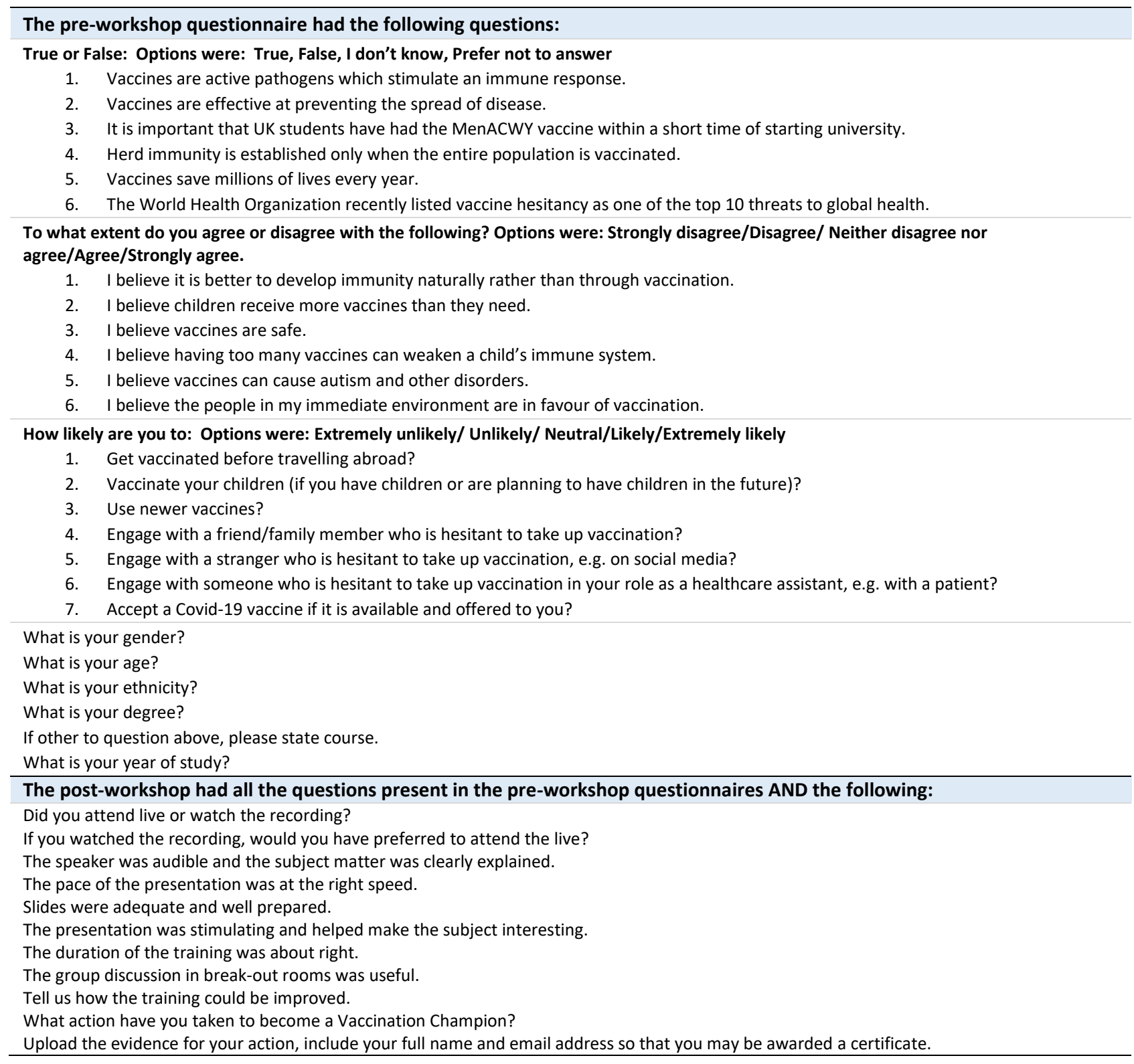

The break-out room session took place prior to the presentation about vaccine hesitancy, and participants in 'rooms' of three to five were asked to discuss what they know about anti-vaccination, such as its prevalence, the possible reasons, and how healthcare practitioners and students can address it.

The online workshop was concluded with a reminder about the vaccination promotion action the participants had to conduct within a week and about the post-workshop questionnaire (questions shown in Table I), which was to be completed within a week of the workshop.

The post-workshop questionnaire contained: (i) the same questions as the pre-training questionnaire (so that any differences brought about by the workshop could be determined), (ii) some additional questions about the workshop itself, such as its clarity, duration, the pace of delivery and how the presentations and the course could be improved in future, and (iii) a section for students to write about the vaccination promotion action they had conducted and to upload evidence of this action, such as a link to their social media posts.

Post-workshop, the authors kept in touch with the participants, for example, with reminders to complete the questionnaire, upload evidence of their action if that was missing, and with links to existing resources about vaccination promotion and upcoming webinars. A few months after the workshop, a follow-up session was held via zoom. This had not been planned when the course structure was decided but was added in response to feedback from the participants. The vaccination champions were also emailed certificates of their participation in this course. 


\section{Results}

\section{Demographics of participants}

Seventy-two participants, mostly from UCL, registered for the course. While the course was only advertised by email to MPharm and MSc. students at UCL School of Pharmacy, three registrants were from other universities, namely the University of California San Francisco (UCSF), the University of Southampton and Queen Mary University of London (QMUL), studying Nursing (UCSF) or Medicine (Southampton, QMUL) and had found the course through Eventbrite. Seventy participants completed the pre-workshop questionnaires, of whom 53 (76\%) attended the workshop, conducted a vaccination promotion action, and completed the post-training questionnaire. The sample was skewed towards female and Black, Asian and Minority Ethnic (BAME) participants, with secondyear MPharm undergraduates making up the bulk. The demographic characteristics of the participants are shown in Table II.

Table II: Demographic characteristics of participants

\begin{tabular}{|c|c|c|c|}
\hline & & $\begin{array}{l}\text { Pre-Workshop } \\
\text { questionnaire }(n=70)\end{array}$ & $\begin{array}{l}\text { Post-workshop } \\
\text { questionnaire }(n=53)\end{array}$ \\
\hline \multirow[t]{3}{*}{ Gender } & Female & $59(84 \%)$ & $43(81 \%)$ \\
\hline & Male & $11(16 \%)$ & $10(19 \%)$ \\
\hline & Total & $70(100 \%)$ & $53(100 \%)$ \\
\hline \multirow[t]{2}{*}{ Age (years) } & & $18-43$ (mean 22, sd 4) & $18-26$ (mean 21, SD 2) \\
\hline & Total & (70) $100 \%$ & 52 (98\%); 1 missing \\
\hline \multirow[t]{6}{*}{ Ethnicity } & Asian or Asian British & $33(47 \%)$ & $26(49 \%)$ \\
\hline & Black/African/Caribbean/Black British & $10(14 \%)$ & $7(13 \%)$ \\
\hline & Mixed Ethnic Group & $4(6 \%)$ & $4(8 \%)$ \\
\hline & White & $6(9 \%)$ & $5(9 \%)$ \\
\hline & Other & $17(24 \%)$ & $11(21 \%)$ \\
\hline & Total & 70 (100\%) & $53(100 \%)$ \\
\hline \multirow[t]{3}{*}{ University } & UCL & 69 (99\%) & $53(100 \%)$ \\
\hline & UCSF & $1(1 \%)$ & 0 \\
\hline & Total & $70(100 \%)$ & $53(100 \%)$ \\
\hline \multirow[t]{4}{*}{ Degree } & MPharm Pharmacy & $57(81.4 \%)$ & $48(91 \%)$ \\
\hline & MSc & $12(17.1 \%)$ & $5(9 \%)$ \\
\hline & Nursing & $1(1.4 \%)$ & 0 \\
\hline & Total & 70 (100\%) & $53(100 \%)$ \\
\hline \multirow[t]{6}{*}{ Year of Study } & First & $12(17 \%)$ & $8(15 \%)$ \\
\hline & Second & $30(43 \%)$ & $28(53 \%)$ \\
\hline & Third & $13(19 \%)$ & $9(17 \%)$ \\
\hline & Fourth & $7(10 \%)$ & $3(6 \%)$ \\
\hline & Post-graduate & $8(11 \%)$ & $5(9 \%)$ \\
\hline & Total & $70(100 \%)$ & $53(100 \%)$ \\
\hline \multirow{3}{*}{$\begin{array}{l}\text { Attended the } \\
\text { workshop life or } \\
\text { watched the } \\
\text { recording }\end{array}$} & Live & & $50(94 \%)$ \\
\hline & Recording & & $3(6 \%)$ \\
\hline & Total & Not available & $53(100 \%)$ \\
\hline
\end{tabular}

\section{Online workshop and responses to Slido polls}

The two-hour online workshop, attended by 53 participants and facilitated by three organisers (the authors), comprising a presentation, four Slido polls, two break-out room sessions, and with the chat function seemed to be of appropriate duration and enabled audience participation and interaction. The Slido polls were completed by most participants, with extensive answers to the text questions. When participants were asked how confident they were about promoting vaccination on a scale of 1-10 (with 10 being very confident), the scores ranged from 2 to 10 , with the majority (61\% of 48 responses) selecting 5 to 7. There were 37 responses to the Slido poll 'What have you learned about vaccination and vaccines that you did not know before?' Of particular note among the wide range of responses was a surprise that some members of the public have concerns about vaccination, the realisation of the role of healthcare practitioners in addressing vaccine hesitancy and the learning about the indirect benefits of vaccination, e.g.

'I have just realised the severity of the misconception of vaccines. As a healthcare professional and being in a family who has supported vaccines throughout my life; it is very surprising to see how many people have [SIC] concerns about it.'

'I have learned that educating patients is really important and it may be life-changing for them',

'I learned about the benefits behind vaccination such as economical ones which I haven't heard about before'. 
There were 33 responses to the Slido poll, 'What are the barriers, if any, to you of becoming a vaccination champion?', including five stating that there were no barriers. Other responses indicated barriers related to not knowing vaccine-hesitant people, not knowing how to approach vaccine-hesitant people, not having sufficient knowledge, confidence and communication skills, unsure of reputable sources of information, lack of access to reputable sources, lack of time, being vaccinehesitant themselves especially regarding vaccination against Covid-19, their own 'culture, beliefs, religion and thoughts', 'possibly my ethnicity, culture and familial background', 'Not having a social media platform with anti-vaccination viewers. Therefore, posting on personal social media will reach those with similar beliefs to yours. There is a potential for information to not reach the intended anti-vaccination audience', 'believing that everyone has their own opinion and it should be respected, I find it difficult to engage in a full conversation to persuade them otherwise'.

There were 37 responses to the Slido poll 'What action might you take in the next week regarding the next step for you to become a Vaccination Champion?' and the responses included investigating currently used methods of vaccination promotion and examining their effectiveness, identifying reputable sources 'that can be shared for information on vaccines', learning more about vaccination and vaccines' mechanisms of action to have 'well researched evidence', listening to vaccine-hesitant people about their experiences and views 'so that I can form a more targeted and sensitive approach when addressing them and use those insights to post on social media', understanding whether their parents' culture supports vaccination, talking to family, friends, colleagues and others, some of whom are sceptical and 'convince them to change their views through a two way conversation rather than completely taking charge', blogging to 'encourage vaccine-hesitant people to engage and start a discussion', creating a poster/infographic/information pack/powerpoint, posting on social media to raise awareness, promote the efficacy of vaccines, share the information learnt during the workshop and 'get people to talk about vaccines', to posting 'my vaccination champion certificate on my social media platforms and my Linkedln and inform people of what I learnt and encourage them to become a vaccination champion!'. Promotion on social media was a popular response as "I feel it is the best way to raise awareness and get the information across in this day and age'.

\section{Analysis of pre-and post-workshop questionnaires}

Pre-workshop questionnaires

The pre-workshop questionnaires showed that attendees had a certain level of knowledge about vaccines, and 39 of 70 participants answering at least four of the six knowledge questions correctly. Most attendees correctly answered about vaccine efficacy at preventing the spread of disease (66 out of 70; 94\%) and about vaccines saving millions of lives every year (65 out of $70 ; 93 \%)$. Fewer correctly answered about the nature of vaccines (46 out of $70 ; 66 \%$ ), the meaning of herd immunity (56 out of $70 ; 80 \%$ ), the World Health Organisation listing vaccine hesitancy as one of the top ten threats to global health (35 out of $70 ; 50 \%$ ), and of the need for university students to get vaccinated against meningitis if they were not already vaccinated (50 out of $70 ; 71 \%$ ).

Over half (40 out of $70 ; 57 \%$ ) of participants believed that people in their immediate environment are in favour of vaccination. The pre-workshop questionnaire responses also showed that overall, the attendees held few misconceptions about vaccination, with $76 \%$ (53 out of 70 ) agreeing or strongly agreeing that vaccines are safe and the majority ( $\geq 70 \%$ ) disagreed or strongly disagreed that children receive more vaccines than they need (56 out of $70 ; 80 \%$ ), that it is better to develop immunity naturally rather than through vaccination (49 out of $70 ; 70 \%$ ), that too many vaccines can weaken a child's immune system (50 out of 70 ; $70 \%)$ and that vaccines can cause autism and other disorders (54 out of 70; 77\%). Despite some misconceptions held, the pre-workshop questionnaires showed that the great majority of participants are likely/extremely likely to vaccinate their current/future children (67 out of 70; 96\%), encourage family members to get the recommended vaccines (64 out of 70; 91\%) and get themselves vaccinated before travelling abroad (56 out of $70 ; 80 \%$ ). The preworkshop questionnaires also showed a considerable proportion of participants to be unlikely/extremely unlikely to accept newer vaccines (31 out of 70; 44\%), accept COVID-19 vaccines (27 out of $70 ; 39 \%$ ), to engage with vaccine-hesitant: family/friends (15 out of $70 ; 21 \%$ ); strangers (45 out of $70 ; 64 \%$ ); patients, i.e. in their role as a healthcare practitioner (11 out of 70 ; $16 \%)$.

\section{Post-workshop questionnaires}

Of the 53 post-workshop questionnaires, only 50 could be linked to the corresponding pre-workshop questionnaires, and therefore only these 50 pre-and post-workshop questionnaires were analysed when the influence of the workshop, if any, was explored as below.

Attendance at the online workshop increased knowledge, and a greater proportion of students answered the knowledge questions correctly. Postworkshop, all the 50 responses about vaccine efficacy at preventing disease spread and about vaccines saving 
millions of lives were correct (up from 48 and 46 preworkshop respectively). However, there is room for improvement; in particular, it is noted that the nature of vaccines and of herd immunity needs to be explained and discussed in greater detail as the related questions were correctly answered by only 38 (up from 35 preworkshop) and 44 (up from 42) of the respondents respectively. Similarly, the number of correct answers about the need for UK University students to get the MenACWY vaccine was 44 (up from 39), and the WHO recently listing vaccine hesitancy as one of the top 10 threats to global health was 44 (up from 25).

Attendance at the online workshop reduced (but did not eliminate) misconceptions about vaccination. More students agreed that vaccines are safe (from 40 to 47) and disagreed or strongly disagreed that: it is better to develop immunity naturally than through vaccination (from 38 to 45 ), children receive more vaccines than they need (from 42 to 44 ), having too many vaccines can weaken a child's immune system (from 36 to 45), vaccines can cause autism and other disorders (from 37 to 46). Despite some residual concerns about vaccination following the workshop, although all the participants said they would vaccinate their current/future children and more participants were likely to: get themselves vaccinated before travelling abroad (from 42 to 44 ), use newer vaccines (from 26 to 34); accept a Covid-19 vaccine if it is available and offered to them (from 30 to 40); engage with a friend/family member who is vaccine-hesitant (from 38 to 48); engage with a stranger who is vaccinehesitant (from 20 to 41) and engage with someone who is vaccine-hesitant in their role as a healthcare professional (from 44 to 49 ).

\section{Feedback about the workshop}

Twenty-seven to twenty-eight students gave feedback about the workshop, which was mostly complimentary, with $80-100 \%$ of the attendees agreeing or strongly agreeing that the speaker was audible and the subject matter was clearly explained (28 out of 28 ), the pace was at the right speed ( 25 out of 28 ), the slides were adequate and well-prepared (27 out of 28), the presentation was stimulating and helped make the subject interesting (25 out of 28), the duration was about right (22 out of 27), the group discussion in break-out rooms was useful (20 out of 25).

When asked about what could be improved in future sessions, the suggestions included: (i) increasing the content, such as about current vaccines and their use, COVID-19 vaccines, how vaccines work, statistics of vaccination programs, controversies, reasons for vaccine hesitancy and how to tackle vaccine hesitancy as a healthcare practitioner; (ii) follow-up sessions to discuss challenges faced when acting as vaccination champions; (iii) having a facilitator in break-out rooms, or increasing the numbers of participants in break-out rooms; (iv) including training on promoting vaccination on social media; (v) including a quiz at the end of the workshop for participants to test their knowledge and (vi) sending resources to participants about latest vaccine developments to keep them updated.

\section{Actions taken by participants following the workshop}

Post-workshop, the participants had in-person conversations with friends, parents, siblings, cousins, other family members, neighbours, and customers in a pharmacy and/or promoted vaccination on social media platforms such as Instagram, Twitter, Facebook, YouTube, WordPress, Medium, sharing leaflets, articles, posters they created (Table III). Topics of inperson and online conversations ranged from the direct and indirect benefits of vaccination, vaccine myths and facts, vaccine efficacy and safety, including the very low incidence of adverse effects, herd immunity, MenACWY, Human Papillomavirus (HPV) and (Measles Mumps and Rubella) MMR vaccines, the development of Covid-19 vaccines and the fact that vaccines are rigorously tested prior to being marketed. Participants almost always urged their audience to use reputable sources of information about vaccination, e.g. their general practitioner and on their social media posts, provided links to, for example, the National Health Service (NHS), the WHO, Centre for Disease Control (CDC), The Wellcome Trust, Public Health England and UNICEF websites, and asked their followers to send them questions or comments, and a few followers did ask for more discussion. The social media posts had high levels of engagement; for example, one Instagram post was seen by more than 100 people and many posts were liked, shared and re-shared, and commented upon. One student created several polls on Instagram asking their followers some questions about vaccination (which were answered by over 50 people) and followed up with vaccination facts using WHO links.

Under half of the actions taken were related to inperson conversations, with the other half being on social media platforms (Table III), and many participants reported deep and interactive engagement with their audience. One student reported that their family talked about vaccination for days following the online workshop; another reported that discussions also took place at their sibling's workplace following the discussion at home. It is noteworthy that several students engaged with people they knew to be vaccine-hesitant and that at least three of these subsequently changed their minds, and several decided to conduct more research into vaccination. A selection of the evidence of their actions that course participants uploaded to the post-workshop 
questionnaire can be seen in Appendices A to C. For examples of leaflets and blog posts, please contact the authors directly.

Table III: Number of participants who engaged with actions

\begin{tabular}{|c|c|c|}
\hline Action & $\begin{array}{l}\text { Number of } \\
\text { carried out }\end{array}$ & $\begin{array}{l}\text { tudents who } \\
\text { the actions }\end{array}$ \\
\hline $\begin{array}{l}\text { Conversation with a friend or } \\
\text { family member }\end{array}$ & 18 & \\
\hline $\begin{array}{l}\text { Conversation with patients at } \\
\text { pharmacy placement }\end{array}$ & 1 & \\
\hline \multirow[t]{5}{*}{ Social media post } & $\begin{array}{l}\text { Social } \\
\text { media } \\
\text { platform }\end{array}$ & $\begin{array}{l}\text { Number of } \\
\text { students who } \\
\text { posted on: }\end{array}$ \\
\hline & Instagram & 7 \\
\hline & Twitter & 7 \\
\hline & Facebook & 4 \\
\hline & Youtube & 1 \\
\hline Blog on WordPress & \multicolumn{2}{|l|}{1} \\
\hline $\begin{array}{l}\text { Created a Poster/ Leaflet and } \\
\text { shared it on Social media }\end{array}$ & \multicolumn{2}{|l|}{8} \\
\hline Wrote an article on Medium.com & \multicolumn{2}{|l|}{1} \\
\hline
\end{tabular}

\section{Follow-up session}

An hour follow-up session was held via Zoom 2.5 months after the online workshop. Although all the course participants were invited, only seven joined the session. The small number could be due to the fact that the new academic year had already started and students had other priorities and because of the duration of the gap between the online training and the follow-up. Nevertheless, the participants were very enthusiastic, and the discussion was robust. The authors discussed the actions taken by the participants, and what became apparent was the depth of in-person and online interactions that took place among the participants and their interlocutors, and that Covid-19 was a regular topic in the conversation, in particular how vaccines against Covid-19 are being developed and produced. When the future of the course and how to improve it was discussed, increasing the content, especially regarding debunking vaccine myths, was one theme, and participants unanimously agreed that the vaccination champion course should be part of the degree curriculum rather than an optional activity.

\section{Discussion}

The pre-workshop questionnaires showed that attendees had a medium level of knowledge about vaccines and vaccination prior to the online workshop.
This reflected their scientific background as all the attendees were studying pharmaceutical sciences, medicine, or nursing. The gaps in their knowledge were also expected as most of the pharmaceutical sciences attendees (MPharm Year two students) had not yet studied infection and vaccines in their degree programme. At UCL School of Pharmacy, this theme is covered in the third year of the degree programme, and the authors were considering whether the vaccination champion course should be conducted towards the end of that theme so that armed with broader and deeper scientific knowledge, the vaccination champions feel more confident about promoting vaccination. At the same time, it is expected that the vaccination champion course would increase interest in the scientific study of infection and immunity as this theme acquires greater and immediate relevance.

The fact that 20 out of 70 pre-workshop respondents seemed to be unaware of the need for university students to get vaccinated against meningitis if they had not already got them was somewhat (but not totally) surprising and reflected similar recall rates about the MenACWY in other UCL students (Jones et al., 2020). Getting meningitis (ACWY) and MMR vaccinations (if students have not already done so) is on the list of essential things to do on arrival to university as university students would have seen this information at the start of their course, i.e. nine months or more prior to completing the questionnaire. It seems this information was forgotten by many students.

During the workshop, the topics discussed were vaccines and vaccination, direct and indirect benefits of vaccination, herd immunity, vaccine myths and facts, vaccine hesitancy, and how to address vaccine hesitancy. These topics were selected to cater for the audience's expected knowledge (and gaps in their knowledge) at the time of the course and to prepare the future vaccination champions to discuss and answer questions when they went about promoting vaccination to their families, friends, neighbours, and others.

Attending the workshop increased the attendees' knowledge, as hoped. The less than $100 \%$ success rate for the questions about the nature of vaccines and the meaning of herd immunity could also be due to the fact that most of the attendees had not yet covered infection, immunology, and vaccinology in their degree programme where these are taught in detail, in contrast to the online vaccination championship workshop, where a broad range of topics was covered in two hours. This also explains the lack of knowledge about vaccines, vaccination, and reputable sources of information, as well as the lack of confidence that some of the attendees stated as barriers to them becoming vaccination champions, and their feedback about 
increasing the content covered, providing more resources to the vaccination champions and having follow-up sessions.

This study also indicated significant vaccine hesitancy in the attendees' immediate environment. Although vaccine hesitancy was not directly measured in the attendees' immediate environment, the attendees' response indicates significant numbers of people in the general population who are vaccine-hesitant in the UK, as reported by Luyten and the authors (2019). Vaccine hesitancy in their immediate environment could also have contributed to participation in the course, and nine attendees were reporting discussion with one or more vaccine-hesitant family/friend/neighbour as their 'action' following the online workshop.

The course participants themselves had some concerns about vaccines. The $76 \%$ (of 70 ) agreeing that vaccines are safe prior to the workshop is lower than the 95\% (of 144) reported by Jones and the authors (2020). This could be due to the very different samples of participants, for example, in terms of ethnicity, with most of the participants being of BAME groups in contrast to the sample in (Jones et al., 2020) where the majority belonged to the White ethnic group. Ethnicity is known to affect vaccination uptake and the perceptions about immunisation in the UK (Marlow et al., 2009; Marlow, 2011; Forster et al., 2017). The fact that attendance at the workshop reduced concerns about vaccination shown by the increased numbers of participants who agreed that vaccines are safe, held fewer misconceptions about vaccines, were more likely to accept vaccines and to engage with vaccine-hesitant family, friends, neighbours, strangers and patients after attending the workshop, shows that the training of university students to become vaccination champions could be one way of increasing awareness and vaccine uptake in ethnic groups that are especially likely to have low vaccine uptake.

The actions that were taken by participants following the workshop, such as conversations with family, friends, neighbours and customers in the pharmacy, in person and/or online, and reports that some vaccinehesitant people did change their minds about vaccination shows the potential of vaccination champions in addressing vaccine hesitancy. The participants' use of social media platforms, giving links to known and trusted sources of information such as the NHS and WHO and inviting engagement to promote vaccination is welcome. Given the large proportion of adults, including parents, in the UK who are exposed to negative messages about vaccination more often than to positive messages on social media, a significant proportion of young people valuing online opinion (Royal Society for Public health Vision, Voice and Practice, 2018) and the disproportionate success of social media in promoting vaccine hesitancy (Puri et al., 2020), it is imperative that current and future healthcare practitioners promote vaccination online, and serve the needs of those who are seeking more information and are susceptible to persuasion. In future courses, including training on the use of social media to promote vaccination by an appropriately qualified trainer was considered, as per feedback by the course participants.

This pilot course was very promising, and some certain limitations were noted, which are as follows. The sample was skewed towards female and Black, Asian and Minority Ethnic (BAME) participants, with 2nd Year pharmacy undergraduates making up the majority. It was also noted that tools that elicit self-reported attainment and behaviour changes are considered to provide weak evaluation evidence and are of variable accuracy (Bhandari \& Wagner, 2006). In this study, however, self-reporting pre-and post-workshop questionnaires were combined, alongside participants writing about their vaccination promotion actions and uploading the related evidence. A future consideration to validate the impact of the course could be to encourage participants to seek anonymised feedback from their target audience. In addition, It was noted that while the use of the online method to deliver the course was useful, there was no opportunity for practising vaccine conversations face to face, which would have helped the students initiate discussions with their family members and friends.

\section{Conclusion and reflections for the future}

The pilot project showed the potential of a vaccination champion training course in promoting vaccination. Despite the short duration of the training and the course participants' lack of detailed knowledge about infectious diseases, immune responses and vaccines, they acquired sufficient information and confidence to engage with their family, friends, neighbours, customers in the pharmacy and online followers about vaccination. It seems that because conducting a vaccination promotion action was a requirement of the course and participants had to send us a summary of their action and attach evidence, the participants felt they had 'permission' and/or a reason to engage with people about vaccination promotion.

The course had a direct effect on the participants themselves, increasing their knowledge and reducing misconceptions and vaccine hesitancy. Given that a large proportion of university students are likely to be future parents, training students regarding vaccination and its promotion, and in particular directing them to reputable sources of information, could be one way of 
reducing their own possible vaccine hesitancy in relation to their future children and increases the likelihood that they will feel sufficiently knowledgeable and confident to promote vaccination to other parents in their social circles.

The pilot course discussed in this paper is being shared with other universities in PharmAlliance. Material and support were provided for a first-year pharmacy student at the Eshelman School of Pharmacy at the University of North Carolina to deliver a course to other students in June 2021. Similarly, the authors are planning to support students at the Faculty of Pharmacy and Pharmaceutical Sciences at Monash University in a few months' time to deliver such a course. At UCL, the authors will run the course again, adapted to this year's situation, for example, regarding the availability of vaccines against Covid-19.. The course has also been expanded to include a speaker who will talk about the psychology of taking vaccines and another speaker who will discuss the various ways NHS Staff have been promoting vaccination, with a view to sharing good practices which could be adopted and adapted by the students. The course has also been opened to students reading other degrees at University College London and at the partners in the ULLA Schools of Pharmacy consortium.

In the future, the vaccination promotion course will be revised and incorporated into the MPharm curriculum, and the course will be offered to students studying other health/life sciences and even non-science subjects, following appropriate adaptations and cocreation, especially for students without a scientific background. The authors believe that anyone can become a vaccination champion regardless of background knowledge. After all, most people need to be vaccinated for the elimination and eradication of infectious disease, and conversations about vaccines, especially against Covid-19, are going to keep on increasing for the foreseeable future. Training students to become vaccination champions will equip them with the resources, and at the very least with reputable sources of information and some knowledge and confidence, to engage in these conversations.

\section{Funding}

The student received a scholarship from the Laidlaw Foundation, which enabled her to conduct this project. The other authors received no financial support for the research, authorship, and/or publication of this article.

\section{References}

Aggarwal, A. (2019). Childhood Vaccine Refusal and Hesitancy -Reasons. Indian Journal of Pediatrics, 86(1), 5-6. https://doi.org/10.1007/s12098-018-2809-7

APhA. (n.d.). APhA-ASP Operation Immunization. APhA. Retrieved December 2020, from

https://www.pharmacist.com/apha-asp-operationimmunization

Bhandari, A., \& Wagner, T. (2006). Self-reported utilization of health care services: Improving measurement and accuracy. Medical Care Research and Review, 63(2), 217235. https://doi.org/10.1177/1077558705285298

Bowers, B.L., Rowe, J.M., \& Stafford, R.A. (2020). Using an Algorithm to Assess Vaccination Among Patients Who Attended a Pharmacy Student-Driven Statewide Free Health Screening. Journal of Pharmacy Practice, 33(4), 425-432. https://doi.org/10.1177/0897190018815367

Bushell, M., Frost, J., Deeks, L., Kosari, S., Hussain, Z., \& Naunton, M. (2020). Evaluation of Vaccination Training in Pharmacy Curriculum: Preparing Students for Workforce Needs. Pharmacy, 8(3), Article 151. https://doi.org/10.3390/pharmacy8030151

Church, D., Johnson, S., Raman-Wilms, L., Schneider, E., Waite, N., \& Sharpe, J.P. (2016). A literature review of the impact of pharmacy students in immunization initiatives. Canadian Pharmacists Journal, 149(3), 153-165. https://doi.org/10.1177/1715163516641133

Ciliberti, R., Bragazzi, N. L., \& Bonsignore, A. (2020). The Implementation of the Professional Role of the Community Pharmacist in the Immunization Practices in Italy to Counteract Vaccine Hesitancy. Pharmacy, 8(3), Article 155. https://doi.org/10.3390/pharmacy8030155

Dube, E., Gagnon, D., MacDonald, N.E., \& Hesitancy, S.W.G. V. (2015). Strategies intended to address vaccine hesitancy: Review of published reviews. Vaccine, 33(34), 4191-4203. https://doi.org/10.1016/j.vaccine.2015.04.041

Dube, E., Laberge, C., Guay, M., Bramadat, P., Roy, R., \& Bettinger, J. (2013). Vaccine hesitancy An overview. Human Vaccines \& Immunotherapeutics, 9(8), 1763-1773. https://doi.org/10.4161/hv.24657

Eskola, J., Duclos, P., Schuster, M., MacDonald, N.E., \& Hesitancy, S.W.G.V. (2015). How to deal with vaccine hesitancy? Vaccine, 33(34), 4215-+. https://doi.org/10.1016/j.vaccine.2015.04.043

Facciola, A., Visalli, G., Orlando, A., Bertuccio, M. P., Spataro, P., Squeri, R., Picerno, I., Di Pietro, A. (2019). Vaccine hesitancy: An overview on parents' opinions about vaccination and possible reasons of vaccine refusal. Journal of Public Health Research, 8(1), 13-18, Article 1436. https://doi.org/10.4081/jphr.2019.1436

Forster, A.S., Rockliffe, L., Chorley, A.J., Marlow, L.A.V., Bedford, H., Smith, S.G., \& Waller, J. (2017). Ethnicityspecific factors influencing childhood immunisation decisions among Black and Asian Minority Ethnic groups in the UK: a systematic review of qualitative research. Journal of Epidemiology and Community Health, 71(6), 544-549. https://doi.org/10.1136/jech-2016-207366 
Goldman, R.D., Yan, T.D., Seiler, M., Cotanda, C.P., Brown, J. C., Klein, E.J., Hoeffe, J., Gelernter, R., Hall, E.J., Davis, L.A., Griffiths, A.M., Mater, A., Manzano, S., Gualco, G., Shimizu, N., Hurt, L.T., Ahmed, S., Hansen, M., Sheridan, D., Ali, S., Thompson, C.G., Gaucher, N., Staubli, G. (2020). Caregiver willingness to vaccinate their children against COVID-19:

Cross sectional survey. Vaccine, 38(48), 7668-7673. https://doi.org/10.1016/j.vaccine.2020.09.084

Gust, D.A., Darling, N., Kennedy, A., \& Schwartz, B. (2008). Parents with doubts about vaccines: Which vaccines and reasons why. Pediatrics, 122(4), 718-725. https://doi.org/10.1542/peds.2007-0538

Hanson, K.E., Koch, B., Bonner, K., McRee, A.-L., \& Basta, N. E. (2018). National Trends in Parental Human Papillomavirus Vaccination Intentions and Reasons for Hesitancy, 20102015. Clinical Infectious Diseases, 67(7), 1018-1026. https://doi.org/10.1093/cid/ciy232

Jarrett, C., Wilson, R., O'Leary, M., Eckersberger, E., Larson, H. J., \& Hesitancy, S.W.G.V. (2015). Strategies for addressing vaccine hesitancy - A systematic review. Vaccine, 33(34), 4180-4190. https://doi.org/10.1016/j.vaccine.2015.04.040

Jones, S., Cortina Borja, M., \& Bedford, H. (2020). "I think meningitis is a virus, while septicaemia might be caused by bacteria." A study of vaccination views, disease awareness and MenACWY and MMR uptake among freshers at a London university. International journal of adolescent medicine and health. https://doi.org/10.1515/ijamh-20190254

Kwok, K.O., Li, K.-K., Wei, W.I., Tang, A., Wong, S.Y.S., \& Lee, S.S. (2021). Editor's Choice: Influenza vaccine uptake, COVID-19 vaccination intention and vaccine hesitancy among nurses: A survey. International journal of nursing studies, 114, 103854-103854.

https://doi.org/10.1016/j.ijnurstu.2020.103854

Lane, S., MacDonald, N.E., Marti, M., \& Dumolard, L. (2018). Vaccine hesitancy around the globe: Analysis of three years of WHO/UNICEF Joint Reporting Form data-2015-2017. Vaccine, 36(26), 3861-3867.

https://doi.org/10.1016/j.vaccine.2018.03.063

Larson, H.J., Jarrett, C., Eckersberger, E., Smith, D.M.D., \& Paterson, P. (2014). Understanding vaccine hesitancy around vaccines and vaccination from a global perspective: A systematic review of published literature, 2007-2012. Vaccine, 32(19), 2150-2159.

https://doi.org/10.1016/j.vaccine.2014.01.081

Lazarus, J.V., Ratzan, S.C., Palayew, A., Gostin, L.O., Larson, H. J., Rabin, K., Kimball, S., El-Mohandes, A. (2020). A global survey of potential acceptance of a COVID-19 vaccine. Nature Medicine. https://doi.org/10.1038/s41591-02001226-0

Leask, J., Willaby, H.W., \& Kaufman, J. (2014). The big picture in addressing vaccine hesitancy. Human Vaccines \& Immunotherapeutics, 10(9), 2600-2602. https://doi.org/10.4161/hv.29725

Lee, L., Peterson, G.M., Naunton, M., Jackson, S., \& Bushell, M. (2020). Protecting the Herd: Why Pharmacists Matter in Mass Vaccination. Pharmacy (Basel, Switzerland), 8(4). https://doi.org/10.3390/pharmacy8040199
Lisenby, K.M., Patel, K.N., \& Uichanco, M.T. (2019). The Role of Pharmacists in Addressing Vaccine Hesitancy and the Measles Outbreak. Journal of Pharmacy Practice, Article 0897190019895437.

https://doi.org/10.1177/0897190019895437

Luyten, J., Bruyneel, L., \& van Hoek, A.J. (2019). Assessing vaccine hesitancy in the UK population using a generalized vaccine hesitancy survey instrument. Vaccine, 37(18), 2494-

2501. https://doi.org/10.1016/j.vaccine.2019.03.041

MacDonald, N.E., \& Hesitancy, S.W.G.V. (2015). Vaccine hesitancy: Definition, scope and determinants. Vaccine, 33(34), 4161-4164.

https://doi.org/10.1016/j.vaccine.2015.04.036

Marlow, L.A.V. (2011). HPV vaccination among ethnic minorities in the UK: knowledge, acceptability and attitudes. British Journal of Cancer, 105(4), 486-492. https://doi.org/10.1038/bjc.2011.272

Marlow, L.A.V., Wardle, J., \& Waller, J. (2009). Attitudes to HPV vaccination among ethnic minority mothers in the UK An exploratory qualitative study. Human Vaccines, 5(2), 105110. https://doi.org/10.4161/hv.5.2.7368

McRee, A.-L., Gilkey, M.B., \& Dempsey, A.F. (2014). HPV Vaccine Hesitancy: Findings From a Statewide Survey of Health Care Providers. Journal of Pediatric Health Care, 28(6), 541-549.

https://doi.org/10.1016/j.pedhc.2014.05.003

Nowak, G.J., Gellin, B.G., MacDonald, N.E., Butler, R., \& Hesitancy, S.W.G.V. (2015). Addressing vaccine hesitancy: The potential value of commercial and social marketing principles and practices. Vaccine, 33(34), 4204-4211. https://doi.org/10.1016/j.vaccine.2015.04.039

Oscarsson, M.G., Dahlberg, A., \& Tyden, T. (2011). Midwives at youth clinics attitude to HPV vaccination and their role in cervical cancer prevention. Sexual \& Reproductive Healthcare, 2(4), 137-142.

https://doi.org/10.1016/j.srhc.2011.09.001

Palamenghi, L., Barello, S., Boccia, S., \& Graffigna, G. (2020). Mistrust in biomedical research and vaccine hesitancy: the forefront challenge in the battle against COVID-19 in Italy. European journal of epidemiology. https://doi.org/10.1007/s10654-020-00675-8

Paterson, P., Chantler, T., \& Larson, H. J. (2018). Reasons for non-vaccination: Parental vaccine hesitancy and the childhood influenza vaccination school pilot programme in England. Vaccine, 36(36), 5397-5401.

https://doi.org/10.1016/j.vaccine.2017.08.016

Picchio, C.A., Carrasco, M.G., Sague-Vilavella, M., \& Rius, C. (2019). Knowledge, attitudes and beliefs about vaccination in primary healthcare workers involved in the administration of systematic childhood vaccines, Barcelona, 2016/17. Eurosurveillance, 24(6), 20-29, Article 1800117. https://doi.org/10.2807/1560-7917.es.2019.24.6.1800117

Pogue, K., Jensen, J.L., Stancil, C.K., Ferguson, D.G., Hughes, S.J., Mello, E.J., Burgess, R., Berges, K.B., Quaye, A., Poole, B.D. (2020). Influences on Attitudes Regarding Potential COVID-19 Vaccination in the United States. Vaccines, 8(4), Article 582. https://doi.org/10.3390/vaccines8040582 
Poudel, A., Lau, E.T.L., Deldot, M., Campbell, C., Waite, N.M., \& Nissen, L.M. (2019). Pharmacist role in vaccination: Evidence and challenges. Vaccine, 37(40), 5939-5945. https://doi.org/10.1016/j.vaccine.2019.08.060

Prescott, W.A., \& Bernhardi, C. (2019). Immunization Education in US Pharmacy Colleges and Schools. American Journal of Pharmaceutical Education, 83(5), Article 6765.

Puri, N., Coomes, E.A., Haghbayan, H., \& Gunaratne, K. (2020). Social media and vaccine hesitancy: new updates for the era of COVID-19 and globalized infectious diseases. Human Vaccines \& Immunotherapeutics. https://doi.org/10.1080/21645515.2020.1780846

Royal Society for Public health Vision, Voice and Practice. (2018). Moving The needle: Promoting vaccination uptake across the life course. Available at:

https://www.rsph.org.uk/static/uploaded/3b82db00-a7ef494c-85451e78ce18a779.pdf

Swaney, S.E., \& Burns, S. (2019). Exploring reasons for vaccine-hesitancy among higher-SES parents in Perth, Western Australia. Health Promotion Journal of Australia, 30(2), 143-152. https://doi.org/10.1002/hpja.190
Taylor, S., Landry, C.A., Paluszek, M.M., Groenewoud, R., Rachor, G.S., \& Asmundson, G.J.G. (2020). A Proactive Approach for Managing COVID-19: The Importance of Understanding the Motivational Roots of Vaccination Hesitancy for SARS-CoV2. Frontiers in Psychology, 11, Article 575950. https://doi.org/10.3389/fpsyg.2020.575950

Verger, P., Fressard, L., Collange, F., Gautier, A., Jestin, C., Launay, O., Raude, J., Pulcini, C., Peretti-Watel, P. (2015). Vaccine Hesitancy Among General Practitioners and Its Determinants During Controversies: A National Crosssectional Survey in France. Ebiomedicine, 2(8), 891-897. https://doi.org/10.1016/j.ebiom.2015.06.018

World Health Organization (WHO) (2014). Report of the SAGE Working Group on Vaccine Hesitancy. Available at: https://www.who.int/immunization/sage/meetings/2014/o ctober/1_Report_WORKING_GROUP_vaccine_hesitancy_fin al.pdf

Yaqub, O., Castle-Clarke, S., Sevdalis, N., \& Chataway, J. (2014). Attitudes to vaccination: A critical review. Social Science \& Medicine, 112, 1-11.

https://doi.org/10.1016/j.socscimed.2014.04.018

\section{Appendices}

\section{Appendix A: A conversation with a hesitant vaccine person}

Emma (name changed)

As Emma is part of the Jehovah witness, they are prohibited from receiving vaccinations due to the belief that vaccinations violate the covenant made between God and man. Although the decision as to whether or not they can take vaccines is up to the individual themselves, this was a decision she made for herself and her family.

I then asked her if there were any other reasons that discouraged her from becoming vaccinated. She then explained that she also has a fear of suffering from an allergic reaction due to someone she knows experiencing the exact same thing. It was moderate but still sparked up her scepticism.

It was made apparent from the conversation, that in her opinion this concept of not being vaccinated at all greatly outweighed the potential health risks that could arise following a vaccination. It was also made known from the conversation that she has never been properly educated about the benefits of vaccines or even understood - in appropriate depth - its action. I believe this was due to the difference in curriculum, what was taught (and what wasn't taught) back then and the development of knowledge in relation to vaccines, the advantages, disadvantages which would lead to a more informed decision.

Multiple misconceptions about vaccines did emerge from the conversation, such as her belief that vaccinations contributed to autism or simply the safety concerns she possessed. However, she expressed the fact that she was open to additional information from reliable sources. The information that is readily available is usually difficult to comprehend therefore I believe is it a health professional's responsibility to filter and translate such harder concepts and allow it to be more patient friendly and without jargon. 


\section{Appendix B: Vaccine hesitancy discussion}

I knew my mother was hesitant to have vaccinations due to the misinformation she had read online and from her friends. She would always refer to anecdotes describing the lifethreatening effects of vaccines and solely used these stories to defend her stance on antivaccination. I explained to her that these scenarios are often not well documented and lack a thorough, reproducible testing technique. I further explained that vaccines, as with all medicines, undergo strictly regulated preclinical and clinical trials before being used in the general population. I advised her to look to reputable sources when assimilating medical information, such as the NHS and WHO websites, and to be more critical of new information she may hear from friends or find on her group-chats.

During our discussion, my mother raised the point that harmful side effects were a contributing factor to her anti-vaccination defence. Being aware that she had no known allergies, I explained that the likelihood of her, or a majority of the population, experiencing such a reaction was minimal. I added that the demographic who cannot be vaccinated (due to contraindications e.g. allergies) must therefore be protected via herd immunity in order to reduce the spread of the pathogen. Following our discussion, my mother was intrigued to learn more about vaccines, and I directed her to the WHO website were should could further her knowledge on the subject.

\section{Appendix C: Vaccination champion promotion}

In order to raise awareness and clear up common misconceptions surrounding vaccinations I decided to promote vaccination on my social media. I also gathered people's views and opinions on vaccinations in general and their safety. This helped me to understand the views of people around me and their perception on this topic. Following this, I planned to follow up on the people who viewed vaccinations negatively and engage in a conversation with them. I also shared posters and facts on my social media to educate others around me and widen their knowledge, in particular targeted at people that thought vaccinations are unsafe and given too much to children. Below I have inserted evidence of how I promoted this on my instagram page.
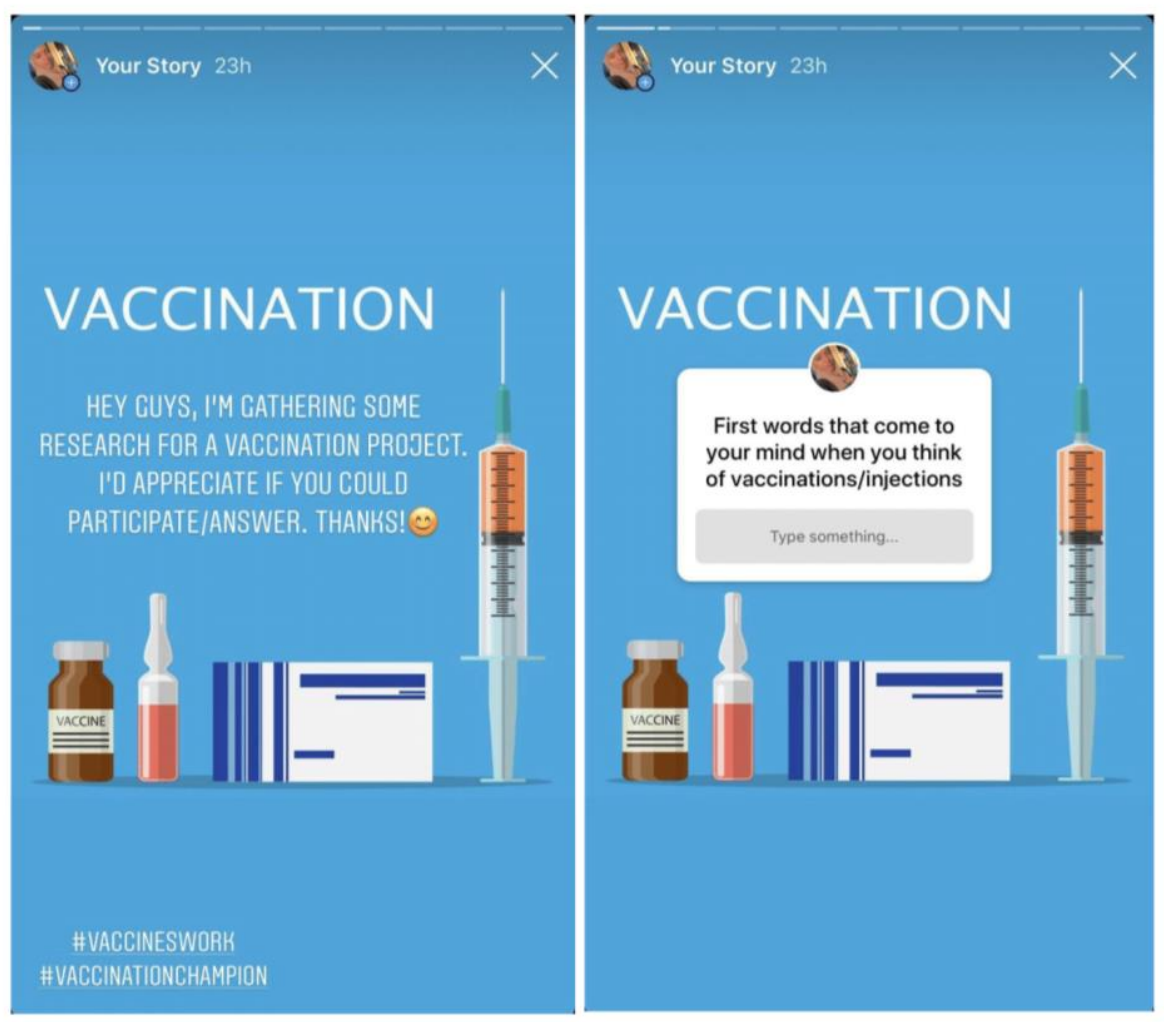